\title{
Meeting report \\ Keystone symposium: The role of microenvironment in tumor induction and progression, Banff, Canada, 5-10 February 2005
}

Jamie L Bascom and Paraic A Kenny

Life Sciences Division, Lawrence Berkeley National Laboratory, University of California, Berkeley, CA 94720, USA

Corresponding author: Paraic A. Kenny, pakenny@lbl.gov

Published: 6 April 2005

This article is online at http://breast-cancer-research.com/content/7/3/113

(c) 2005 BioMed Central Ltd
Breast Cancer Research 2005, 7:113-118 (DOI 10.1186/bcr1030)

detailing crucial interactions between epithelial and stromal cells during both normal mammary development and tumorigenesis. She described a series of wild-type/knockout tissue recombination experiments used to dissect the requirements for epithelially and stromally expressed growth factors and receptors in mammary gland development. In a lecture that was both visually and intellectually stimulating, she showed that intravital imaging technology is a valuable tool with which to interrogate the interactions between stromal and epithelial cells in tumorigenesis. Time-lapse fluorescent videos using mouse mammary tumor virus-polyoma middle $\mathrm{T}$ antigen (MMTV-PyMT) transgenic mice bearing various fluorescent markers demonstrated the dynamic interactions between the tumor and host cellular components.

In the second keynote address, Joan Massague (Memorial Sloan Kettering Cancer Center, New York), described a series of experiments that probed the nature of pre-metastatic cells. Cells from primary tumors in specific organs tend to have a tropism for successful metastasis to distant sites (e.g. breast cancer to bone, lung, liver, and brain; and colon cancer to liver). To what extent are these preferences prespecified in the primary tumor cell population? To address this question, Massague used a metastatic breast cancer cell line, MDA-MB-231, and used nude mice as a 'cell sorter' by introducing cells and collecting and testing those that established metastatic colonies in particular organs. He showed that this cell line is indeed composed of subpopulations with distinct metastatic characteristics that correlate with particular transcriptional profiles [1]. He has begun to analyze the functional relevance of these correlations in overexpression and small interfering RNA (siRNA) studies.
Zena Werb (University of California, San Francisco) set the scene for the meeting with a wide ranging plenary lecture

$\mathrm{CSF} 1$ = colony stimulating factor $1 ; \mathrm{ECM}=$ extracellular matrix; ES = embryonic stem; HGF = hepatocyte growth factor; HIF- $1 \alpha=$ hypoxia inducible factor $1 \alpha$; MMP = matrix metalloproteinase; MMTV = mouse mammary tumor virus; MSF1 = migration stimulating factor 1 ; PyMT = polyoma middle T antigen; SF/HGF = scatter factor/hepatocyte growth factor; siRNA = small interfering RNA; TGCT = testicular germ cell tumor; $\mathrm{TGF} \beta=$ transforming growth factor $\beta$; TGF $\beta R 2=$ transforming growth factor $\beta$ receptor 2 ; TNC $=$ tenascin $\mathrm{C} ; \mathrm{TSP}-1=$ thrombospondin- 1 ; VEGF $=$ vascular endothelial growth factor.

\begin{abstract}
\end{abstract}
Skiers and snowboarders among the delegates were bless stampede for the ski buses each day at $11 \mathrm{am}$. The quality of the meeting was perhaps best reflected by an equally urgent stampede back to the conference center in time for the evening sessions and posters. 


\section{Angiogenesis and microenvironment}

The Monday morning session focused on the role of the microenvironment during angiogenesis. The speakers described the regulation of angiogenesis, both by transcriptional programs and by extracellular pro- and antiangiogenic factors, and the mechanisms by which tumor angiogenesis might be effectively targeted.

The first speaker, Luisa Iruela-Arispe (University of California, Los Angeles), presented work from her group showing that transgenic overexpression of thrombospondin-1 (TSP-1), an ECM component with anti-angiogenic activity, inhibits angiogenesis and tumor growth in MMTV-Neu mice, and effects a downregulation of matrix metalloproteinase (MMP)-9 and decreased expression of vascular endothelial growth factor (VEGF) [2]. Dr Iruela-Arispe's lab is now working to delineate the different functions of a series of different metalloproteinase cleavage fragments of VEGF in tumor angiogenesis.

Nancy Boudreau (University of California, San Francisco) discussed two homeobox transcription factors, HoxD3 and HoxD10, and their apparently opposing roles in breast cancer (Carrio et al., manuscript submitted). HoxD3, which is not expressed in the normal mammary gland, is upregulated during breast cancer progression and upregulates several ECM remodeling enzymes and angiogenic factors, including VEGF [3]. In contrast, Hox D10, a potent inhibitor of angiogenesis, is highly expressed in the normal gland and is absent in invasive carcinomas [4]. Currently, Boudreau and colleagues are concentrating on the organizational role that Hox D10 plays in the normal mammary gland using threedimensional culture assays.

Rapidly growing tumors are frequently hypoxic, and neoangiogenesis may be stimulated by hypoxia-dependent transcriptional activation. Randall Johnson (University of California, San Diego) described the effect of tissue-specific ablation of hypoxia inducible factor $1 \alpha(\mathrm{HIF}-1 \alpha)$ in endothelial cells. Loss of HIF-1 $\alpha$ blocked neoangiogenesis in in vivo matrigel plug assays and also resulted in impaired wound healing in these mice. Most strikingly, tumor xenografts in these mice were smaller and more necrotic than in controls, resulting from a greatly reduced microvessel density [5].

A short talk given by Gavin Thurston (Regeneron Pharmaceuticals, Tarrytown, NY) provided a summary of the array of drugs in development to target VEGF signaling. He is currently studying the efficacy of a decoy receptor for VEGF, 'VEGF-TRAP', against angiogenesis in vivo and has shown that this treatment causes decreased tumor burden in the RIP-TAG transgenic mouse model.

Mary Hendrix (Children's Memorial Research Center at Northwestern University, Chicago) described an alternative to angiogenesis used by aggressive melanoma cells, termed organize to form ECM-rich fluid conducting ductal networks within the tumor, lined by the tumor cells themselves and connected to the vasculature of the host. This reorganization of the tumor cells facilitates the supply of oxygen and nutrients. Hendrix also showed that these networks are resistant to classical inhibitors of angiogenesis, such as endostatin [6].

\section{Microenvironmental influences on metastasis} Interactions between tumor cells and cellular or proteinaceous components of their microenvironment may inhibit or facilitate metastasis. In this evening session, the speakers discussed some of the signaling events involved and the identification of pro-metastatic transcriptional programs.

Large numbers of tumor-infiltrating macrophages often correlate with poor prognosis. Jeffrey Pollard (Albert Einstein College of Medicine, New York) described his laboratory's work on colony stimulating factor 1 (CSF1), a protein secreted by tumor cells that acts as a potent chemoattractant for macrophages. Knockout of CSF1 attenuated macrophage recruitment in the MMTV-PyMT mammary tumor model, resulting in a delayed progression to metastasis of these tumors. Conversely, tumor progression and metastasis were accelerated by CSF1 overexpression in the same model. Thus, CSF1 is implicated in recruiting these VEGF, MMP, and protease secreting pro-angiogenic immune cells to the tumor, which facilitates tumor progression [7].

Kenneth Pienta (University of Michigan, Ann Arbor) presented gene and protein expression data from primary and metastatic prostate cancers from a series of 32 rapid 'warm' autopsies. While bone represented the most common site of metastasis, non-osseous sites included lymph nodes, liver, lung, and adrenal gland. Metastases to different sites (from the same primary tumor) frequently showed significant differences in histology and gene expression [8]. Thus a single regimen might not reasonably be expected to treat such a heterogeneous disease. Pienta outlined an aggressive multi-targeted chemotherapy approach that he is testing in this patient population.

Using an approach similar to that outlined by Joan Massagué, Richard Hynes (Massachusetts Institute of Technology, Cambridge) isolated lung metastases formed following tail vein injection of melanoma cell lines [9]. Successive isolation and re-injection selected for a highly lung-tropic population. Gene expression analysis revealed several changes compared to the parental population. Hynes discussed in detail his analysis of one such gene, which encodes an orphan G-protein coupled receptor (GPR56) with a role in adhesion signaling, that was downregulated in the metastatic variant. Restoration of expression of this gene, while not altering cell growth rate in vitro, significantly attenuated the metastatic potential of these cells. 
Breast cancer soon after pregnancy is associated with high rates of metastasis and poor survival. Pepper Schedin (University of Colorado Health Sciences Center, Denver) described an analysis of the differing phenotypic effects induced in breast cancer cells by purified extracellular matrices from non-parous, parous, and involuting rat mammary glands. The involution matrix, which was enriched in fibronectin fragments, promoted breast cancer cell invasion and metastasis, whereas the other matrices suppressed these phenotypes. Addition of fibronectin fragments to these other matrices was sufficient to elicit the invasive phenotype [10].

\section{Stromal-epithelial and heterotypic interactions}

This session focused on reciprocal paracrine interactions between stromal and epithelial cells in tumor progression. Using a variety of models, speakers discussed the effects of stromally secreted factors on tumor initiation and progression.

Harold Moses (Vanderbilt University, Nashville) described the complexity of transforming growth factor $\beta$ (TGF $\beta$ ) signaling in cancer progression. Specific ablation of transforming growth factor $\beta$ receptor 2 (TGF $\beta$ R2) in mammary epithelium resulted in lobuloalveolar hyperplasia and, in an MMTV-PyMT background, reduced tumor latency and enhanced metastases. Knockout of TGF $\beta R 2$ in stromal fibroblasts (by crossing with FSP1-Cre) resulted in hyperproliferation of adjacent epithelia and tumorigenesis in several organs, a striking demonstration of the power of stromal changes to effect epithelial transformation. Paracrine stimulation of epithelial cells by stromally produced hepatocyte growth factor (HGF) has been implicated in this phenotype [11].

Leland Chung (Emory University School of Medicine, Atlanta) is investigating the mechanisms by which prostate cancer cells interact with bone stromal cells during osseous metastasis. Chung reported that reciprocal interactions between prostate cancer cells and bone stromal fibroblasts in three-dimensional cultures accentuate the growth and invasion of the tumor cells in vitro and in vivo [12]. In a process termed osteomimicry, these metastatic prostate cancer cells invade normal bone tissue by upregulating bone matrix proteins such as osteocalcin, osteopontin, osteonectin, and bone-sialoprotein. His group determined that a soluble factor secreted by both bone and prostate cancer cells, $\beta 2$ microglobulin, controls the expression of these bone matrix proteins and is upregulated during prostate cancer invasion into bone.

Mary Helen Barcellos-Hoff (Lawrence Berkeley National Laboratory, Berkeley) discussed radiation carcinogenesis in terms of its effects on microenvironment during cancer initiation and progression. Using the mouse mammary gland as a model, she described the effect of ionizing radiation on the mammary stroma and its contribution to neoplastic behavior. The genomic changes that accumulate in tumors forming in irradiated hosts are now being investigated.
Olivier de Wever (University Hospital Ghent, Ghent) is studying the role of the stromal myofibroblasts in cancer invasion. Myofibroblasts from colon cancer tissue promote the invasiveness of colon cancer cells in in vitro assays by secretion of scatter factor/hepatocyte growth factor (SF/ HGF) and tenascin C (TNC), which lead to downregulation of RhoA and upregulation of Rac activity [13]. De Wever and colleagues [14] also showed that TGF $\beta$ secreted by colon cancer cells stimulates invasion by stromal myofibroblasts in vitro in a c-jun amino-terminal kinase (JNK)-dependent manner. Myofibroblast $\mathrm{N}$-cadherin was essential for this process.

\section{Recent advances in signaling in 3D microenvironments}

Researchers are increasingly taking advantage of sophisticated physiological culture systems to model in vivo biological processes.

Joan Brugge (Harvard Medical School, Boston) presented data on her laboratory's extensive analysis of the effect of oncogenes and tumor suppressors on mammary epithelial morphogenesis in a three-dimensional matrigel culture assay. Brugge's lab has transduced MCF10A cells with 1000 cancer-related genes. These cells span the full spectrum of cancer related phenotypes - escape from growth arrest, filling of lumen, loss of tissue polarity, alterations in cell polarity, disruption of cell-cell junctions, and acquisition of invasive behavior. The amenability of these cultured mammary acini to genetic manipulation has allowed Brugge to begin to address the cooperative (and antagonistic) effects between various pathways.

Valerie Weaver (University of Pennsylvania, Philadelphia) explored the role of mechanotransduction in mammary epithelial morphogenesis and transformation. She showed that stiffness (viscoelastic modulus) increases in vivo during progression from the normal to transformed mammary gland. In a series of in vitro three-dimensional culture assays using gels of defined viscoelastic properties, she demonstrated that modulating the stiffness of the microenvironment can indeed alter the phenotype of breast epithelial cells by altering signaling homeostasis, with increasing stiffness leading to increasingly transformed behavior.

John Muschler (California Pacific Medical Center, San Francisco) described his work on the functional characterization of the ECM receptor, dystroglycan, in mammary epithelial cells. His laboratory has previously demonstrated a loss of dystroglycan function in several human breast cancer cell lines. Here, using a conditional knockout of dystroglycan in cultured cells, Muschler and colleagues defined an essential role for dystroglycan in signaling from laminin-1. Recruitment and assembly of laminin-1 to the cell surface required dystroglycan expression, as did subsequent signals from laminin-1 for cell polarity and tissue specific gene expression. 
Edna Cukierman (Fox Chase Cancer Center, Philadelphia) described a three-dimensional culture model system of stroma derived from tumor-associated fibroblasts at various stages of cancer progression. Cell signaling events within these matrices differ from those occurring on plastic substrata. Removal of tumor-associated fibroblasts from these cultures and addition of normal fibroblasts to the matrix resulted in upregulation in these cells of markers associated with tumor fibroblasts.

\section{Cancer models}

In this session, a number of sophisticated cancer models were presented and the implications of the derived data were discussed.

Ronald DePinho (Dana-Farber Cancer Institute, Boston) presented a succession of transgenic mouse models of pancreatic adenocarcinoma that closely reflect the stages of progression seen in the human disease. Using a series of floxed alleles on a PDX-Cre background he delineated a successive requirement for K-Ras activation, Ink4A/Arf loss and p53 loss in pancreatic cancer progression [15]

Elaine Fuchs (Rockefeller University, New York) described her laboratory's characterization of epidermal stem cells. Using transgenic mice with a histone 2B-GFP fusion under the control of a K5-rtTA transgene, Fuchs and colleagues employed a pulse-chase strategy to label these infrequently cycling cells in the skin. These cells, located in the bulge of the hair follicle, are mobilized to proliferate upon wounding or at the start of each new hair cycle. Isolation of these label-retaining cells for further characterization revealed that they form larger colonies in culture than their transitamplifying progeny and they retain the capacity to reconstitute epidermis, sebaceous glands, and hair follicles in transplant experiments $[16,17]$.

Epigenetic changes frequently accompany genomic changes during tumor progression. Rudolf Jaenisch (Massachusetts Institute of Technology, Cambridge) described attempts to generate embryonic stem (ES) cells by transferring tumor cell nuclei to enucleated oocytes. While this process was extremely inefficient, a single ES cell line was obtained from a malignant melanoma nucleus [18]. In a striking parallel with the decades-old work of Beatrice Mintz [19], melanomaderived ES cells gave rise to normal appearing chimeric mice. Despite the subsequent onset of melanomas, these studies provide a remarkable demonstration of the reversibility of the cancer phenotype.

Following up on earlier observations that deregulated expression of MMP-3 in transgenic mice could lead to genomic instability and mammary tumorigenesis [20], Derek Radisky (Lawrence Berkeley National Laboratory, Berkeley) provided detailed insight into the mechanism underpinning cytoskeletal changes that lead to upregulation of reactive oxygen species that are both necessary and sufficient to induce epithelial to mesenchymal transition, oxidative DNA damage, and genomic instability.

The zebrafish offers several advantages as a model organism in cancer biology. Testicular germ cell tumors (TGCTs) in zebrafish share certain similarities with the human disease, such as post-pubertal onset, an apparent premalignant in situ lesion, and sensitivity to radiation treatment. James Amatruda (Southwestern Medical Center, Dallas) and colleagues have identified a highly penetrant, dominantly inherited mutation that causes TGCTs in zebrafish and are using interval haplotype mapping to narrow down the locus and clone the mutated gene.

\section{The extracellular matrix and its degrading enzymes in tumor progression and metastasis} MMPs have long been of interest to cancer biologists for their ability to degrade components of the ECM and to shed cell surface ligands. Lynn Matrisian (Vanderbilt University School of Medicine, Nashville) collaborated with Mitsuru Futakuchi (Universities of Nebraska and Nagoya, Japan) to study prostate cancer growth in the bone microenvironment by transplanting chemically induced prostate cancer cells into the cranium and spines of syngeneic F344 rats. Genes commonly found in human prostate-to-bone metastasis, including PTHrP, RANKL, and OPG, were upregulated. MMP-7 was identified as being highly expressed in osteoclasts in areas of high osteolysis. Matrisian's lab further determined that MMP-7 selectively cleaves and releases a soluble form of RANKL, a potent activator of osteolysis found primarily on the surface of osteoblasts. MMP-7 solubilized RANKL was capable of activating osteoclasts and resorbing dentin discs. Immunodeficient MMP-7-null mice showed a significant decrease in osteolysis compared to controls, suggesting that MMP-7 plays a role in bone resorption during prostate metastasis to bone through solubilization of RANKL.

Raghu Kalluri (Harvard Medical School, Boston) began by describing a 'degradomics' analysis of proteolytic cleavage fragments of extracellular matrix proteins. Of 166 fragments identified, 14 possessed anti-angiogenic activity. He focused on tumstatin, a C-terminal fragment of type IV collagen. Mice lacking the gene encoding this collagen chain experienced enhanced angiogenesis during tumor progression, which was restored to normal by injection with recombinant tumstatin $[21,22]$. Similarly, tumorigenesis was accelerated in mice defective in MMP-9 (which cleaves collagen IV to release tumstatin) on both MMTV-Neu and APCmin backgrounds. Conversely, transgenic mice overexpressing either tumstatin or endostatin were significantly protected from tumorigenesis.

Seth Schor (University of Dundee) presented an interesting talk on the identification of a secreted protein that underlies the fetal fibroblast phenotype described 20 years ago by his 
laboratory. Migration stimulating factor 1 (MSF1) is a splicevariant of fibronectin that produces a truncated isoform that corresponds approximately to the most amino-terminal $70 \mathrm{kDa}$ [23]. Although this isoform is not expressed in normal adult fibroblasts, it is expressed in both tumor-associated and fetal fibroblasts, where it exerts a potent motogenic effect at femtomolar levels. Multiple cell types respond to MSF-1, which may promote malignant potential.

James Quigley (Scripps Research Institute, La Jolla) described a model in which tumor cells in collagen onplants on the chorioallantoic membrane of chick embryos stimulate angiogenesis in an MMP-dependent fashion as well as intravasate and metastasize. Species-specific reagents were used to delineate the contribution of various host and tumor proteins and cell types, with MMP-9 and MMP-13 provided by the chicken heterophils and macrophages, respectively, playing particularly important roles [24].

\section{Role of inflammatory cells and cytokines in the tissue and tumor microenvironment}

A prevailing theme throughout the meeting was the diverse contributions made by cells of the immune system to tumor progression and metastasis. In this session, the speakers used elegant transgenic mouse models to delineate these functions. The session concluded with a presentation detailing the transcriptional profile of each of the cell types in the breast tumor microenvironment.

Luis Parada (University of Texas Southwestern Medical School, Dallas) presented a series of mouse models of brain tumors, both neurofibromas and glioblastomas, resulting from deletion of the tumor suppressor NF1. These models, which closely mimic many aspects of the human disease, also shed light on the role of the heterozygous status of nontumor host cells during malignant progression in patients with this familial cancer syndrome. The outcome of NF1 deletion in schwann cells depended on the status of the NF1 alleles in the adjacent stroma [25]. NF1-/- schwann cells preferentially enhance the proliferation of $\mathrm{NF1}^{+/-}$mast cells which infiltrate the tumor - these in turn enhance tumor growth. In contrast, the onset of $\mathrm{NF}^{-1-}$ tumors was suppressed when the surrounding stromal cells retain both wild-type NF1 alleles.

Douglas Hanahan (University of California, San Francisco) analyzed the contribution of stromal factors to angiogenesis in a mouse cervical carcinoma model in which the HPV16 early region is expressed from the $\mathrm{K} 14$ promoter. Crossing these mice with mice null for MMP-9 resulted in smaller, less vascularized tumors. Expression of MMP-9 by tumorinfiltrating macrophages was implicated in the promotion of angiogenesis. He demonstrated that zoledronic acid treatment can effectively target MMP-9 expression and activity in macrophages, resulting in a reduced interaction between VEGF and its receptor [26].
Using the same K14-HPV16 model of squamous cell carcinogenesis, this time concentrating on the skin, Lisa Coussens (University of California, San Francisco) described a requirement for cells of the adaptive immune system for the recruitment of cells of the innate immune system to the tumor site, where they promote tumor progression by secretion of proteases and cytokines. Crossing these mice onto backgrounds deficient in different lineages of $T$ and $B$ cells allowed her to define a critical requirement for $B$ lymphocytes for mast cell recruitment to the tumor site.

In a transcriptional profiling tour de force, Kornelia Polyak (Dana-Farber Cancer Institute, Boston) prospectively isolated each of the cell types that comprise normal breast tissue, as well as those from both in situ and invasive carcinoma, and determined the gene expression profile of each population using serial analysis of gene expression [27]. Although genetic changes were only detected in tumor epithelial cells, all cell types underwent significant gene expression changes, with frequent alterations detected in genes encoding receptors and secreted proteins. Her subsequent analysis focused on an orphan chemokine, CXCL14, that binds epithelial cells in vivo and in vitro and promotes the migration and invasion of MDA-MB-231 cells in culture.

\section{Concluding session}

The final talk from Judah Folkman (Children's Hospital, Boston) was a fitting conclusion to a most stimulating meeting. Dr Folkman provided both an historical perspective on the field of angiogenesis and an inspiring discussion of the future of therapeutics: drugs that directly block angiogenesis signaling pathways as well as drugs that increase production of endogenous angiogenesis inhibitors. Lastly, he described a model in which different subpopulations of tumor cells are inherently angiogenic and non-angiogenic. Although proliferating at the same rate in vitro, only angiogenesis-competent tumor cells formed tumors in nude mice, whereas their nonangiogenic counterparts formed microscopic dormant growths [28].

The critical contribution of microenvironmental factors to tumor progression is now widely appreciated. Interest in this field is growing and research is now advancing rapidly. We expect that this Keystone symposium will be the first of many more meetings that highlight the perspective of cancer biologists who view cancer as a disease of tissues, as well as of cells.

\section{Competing interests}

The author(s) declare that they have no competing interests.

\section{Acknowledgements}

The attendance of PK at this meeting was supported by a travel fellowship from the National Cancer Institute (1 R13-CA113258-01). The authors are supported by DOD BCRP: DAMD17-03-1-0295 (JLB) and DAMD17-00-1-0224 (PK); and by grants from the OBER office of the $\mathrm{DOE}$ and the $\mathrm{NCl}$ to Mina $J$ Bissell. 


\section{References}

1. Minn AJ, Kang Y, Serganova I, Gupta GP, Giri DD, Doubrovin M, Ponomarev V, Gerald WL, Blasberg R, Massague J: Distinct organ-specific metastatic potential of individual breast cancer cells and primary tumors. J Clin Invest 2005, 115:44-55.

2. Rodriguez-Manzaneque JC, Lane TF, Ortega MA, Hynes RO, Lawler J, Iruela-Arispe ML: Thrombospondin-1 suppresses spontaneous tumor growth and inhibits activation of matrix metalloproteinase- 9 and mobilization of vascular endothelial growth factor. Proc Natl Acad Sci USA 2001, 98:12485-12490.

3. Boudreau N, Andrews C, Srebrow A, Ravanpay A, Cheresh DA: Induction of the angiogenic phenotype by Hox D3. J Cell Biol 1997, 139:257-264.

4. Myers C, Charboneau A, Cheung I, Hanks D, Boudreau N: Sustained expression of homeobox D10 inhibits angiogenesis. Am J Pathol 2002, 161:2099-2109.

5. Tang N, Wang L, Esko J, Giordano FJ, Huang Y, Gerber HP, Ferrara N, Johnson RS: Loss of HIF-1alpha in endothelial cells disrupts a hypoxia-driven VEGF autocrine loop necessary for tumorigenesis. Cancer Cell 2004, 6:485-495.

6. van der Schaft DW, Seftor RE, Seftor EA, Hess AR, Gruman LM, Kirschmann DA, Yokoyama Y, Griffioen AW, Hendrix MJ: Effects of angiogenesis inhibitors on vascular network formation by human endothelial and melanoma cells. J Natl Cancer Inst 2004, 96:1473-1477.

7. Lin EY, Nguyen AV, Russell RG, Pollard JW: Colony-stimulating factor 1 promotes progression of mammary tumors to malignancy. J Exp Med 2001, 193:727-740.

8. Shah RB, Mehra R, Chinnaiyan AM, Shen R, Ghosh D, Zhou M, Macvicar GR, Varambally S, Harwood J, Bismar TA, Kim R, Rubin $\mathrm{MA}$, Pienta $\mathrm{KJ}$ : Androgen-independent prostate cancer is a heterogeneous group of diseases: lessons from a rapid autopsy program. Cancer Res 2004, 64:9209-9216.

9. Clark EA, Golub TR, Lander ES, Hynes RO: Genomic analysis of metastasis reveals an essential role for RhoC. Nature 2000 , 406:532-535

10. Schedin P, Mitrenga T, McDaniel S, Kaeck M: Mammary ECM composition and function are altered by reproductive state. Mol Carcinog 2004, 41:207-220.

11. Bhowmick NA, Chytil A, Plieth D, Gorska AE, Dumont N, Shappell S, Washington MK, Neilson EG, Moses HL: TGF-beta signaling in fibroblasts modulates the oncogenic potential of adjacent epithelia. Science 2004, 303:848-851

12. Hsieh CL, Gardner TA, Miao L, Balian G, Chung LW: Cotargeting tumor and stroma in a novel chimeric tumor model involving the growth of both human prostate cancer and bone stromal cells. Cancer Gene Ther 2004, 11:148-155.

13. De Wever O, Nguyen QD, Van Hoorde L, Bracke M, Bruyneel E, Gespach C, Mareel M: Tenascin-C and SF/HGF produced by myofibroblasts in vitro provide convergent pro-invasive signals to human colon cancer cells through RhoA and Rac. Faseb J 2004, 18:1016-1018.

14. De Wever O, Westbroek W, Verloes A, Bloemen N, Bracke M, Gespach C, Bruyneel E, Mareel M: Critical role of N-cadherin in myofibroblast invasion and migration in vitro stimulated by colon-cancer-cell-derived TGF-beta or wounding. J Cell Sci 2004, 117:4691-4703.

15. Aguirre AJ, Bardeesy N, Sinha M, Lopez L, Tuveson DA, Horner J, Redston MS, DePinho RA: Activated Kras and Ink4a/Arf deficiency cooperate to produce metastatic pancreatic ductal adenocarcinoma. Genes Dev 2003, 17:3112-3126.

16. Tumbar T, Guasch G, Greco V, Blanpain C, Lowry WE, Rendl M, Fuchs E: Defining the epithelial stem cell niche in skin. Science 2004, 303:359-363.

17. Blanpain C, Lowry WE, Geoghegan A, Polak L, Fuchs E: Selfrenewal, multipotency, and the existence of two cell populations within an epithelial stem cell niche. Cell 2004, 118:635-648.

18. Hochedlinger K, Blelloch R, Brennan C, Yamada Y, Kim M, Chin $\mathrm{L}$, Jaenisch R: Reprogramming of a melanoma genome by nuclear transplantation. Genes Dev 2004, 18:1875-1885.

19. Mintz B, Illmensee $\mathrm{K}$ : Normal genetically mosaic mice produced from malignant teratocarcinoma cells. Proc Natl Acad Sci USA 1975, 72:3585-3589.

20. Sternlicht MD, Lochter A, Sympson CJ, Huey B, Rougier JP, Gray JW, Pinkel D, Bissell MJ, Werb Z: The stromal proteinase MMP3/stromelysin-1 promotes mammary carcinogenesis.
21. Sund $M$, Hamano $Y$, Sugimoto $H$, Sudhakar $A$, Soubasakos $M$, Yerramalla U, Benjamin LE, Lawler J, Kieran M, Shah A, Kalluri R: Function of endogenous inhibitors of angiogenesis as endothelium-specific tumor suppressors. Proc Natl Acad Sci USA 2005, 102:2934-2939.

22. Hamano $Y$, Zeisberg M, Sugimoto H, Lively JC, Maeshima Y, Yang C, Hynes RO, Werb Z, Sudhakar A, Kalluri R: Physiological levels of tumstatin, a fragment of collagen IV alpha3 chain, are generated by MMP-9 proteolysis and suppress angiogenesis via alphaV beta3 integrin. Cancer Cell 2003, 3:589-601.

23. Schor SL, Ellis IR, Jones SJ, Baillie R, Seneviratne K, Clausen J, Motegi K, Vojtesek B, Kankova K, Furrie E, Sales MJ, Schor AM, Kay RA: Migration-stimulating factor: a genetically truncated onco-fetal fibronectin isoform expressed by carcinoma and tumor-associated stromal cells. Cancer Res 2003, 63:88278836.

24. Zijlstra A, Aimes RT, Zhu D, Regazzoni K, Kupriyanova T, Seandel M, Deryugina El, Quigley JP: Collagenolysis-dependent angiogenesis mediated by matrix metalloproteinase-13 (collagenase-3). J Biol Chem 2004, 279:27633-27645.

25. Zhu Y, Ghosh P, Charnay P, Burns DK, Parada LF: Neurofibromas in NF1: Schwann cell origin and role of tumor environment. Science 2002, 296:920-922.

26. Giraudo E, Inoue M, Hanahan D: An amino-bisphosphonate targets MMP-9-expressing macrophages and angiogenesis to impair cervical carcinogenesis. J Clin Invest 2004, 114:623633.

27. Allinen M, Beroukhim R, Cai L, Brennan C, Lahti-Domenici J, Huang H, Porter D, Hu M, Chin L, Richardson A, Schnitt S, Sellers WR, Polyak K: Molecular characterization of the tumor microenvironment in breast cancer. Cancer Cell 2004, 6:1732.

28. Achilles EG, Fernandez A, Allred EN, Kisker O, Udagawa T, Beecken WD, Flynn E, Folkman J: Heterogeneity of angiogenic activity in a human liposarcoma: a proposed mechanism for "no take" of human tumors in mice. J Natl Cancer Inst 2001 93:1075-1081. 